\title{
Disruption of urine metabolome and its interaction with gut microbiome by low-dose exposure of glyphosate-based herbicide in a rodent model
}

Jianzhong Hu ( $\sim$ jianzhong.hu@mssm.edu )

Icahn School of Medicine at Mount Sinai https://orcid.org/0000-0001-8546-5111

Corina Lesseur

Icahn School of Medicine at Mount Sinai

Miao Yu

Icahn School of Medicine at Mount Sinai

Fabiana Manservisi

Cesare Maltoni Cancer Research Center

Simona Panzacchi

Ramazzini Institute

Daniele Mandrioli

Ramazzini Institute

Fiorella Belpoggi

Ramazzini Institute

Jia Chen

Icahn School of Medicine at Mount Sinai

Lauren Petrick

Icahn School of Medicine at Mount Sinai

\section{Research}

Keywords: Roundup, Glyphosate, metabolomics

Posted Date: March 10th, 2020

DOl: https://doi.org/10.21203/rs.3.rs-16601/v1

License: (c) (i) This work is licensed under a Creative Commons Attribution 4.0 International License.

Read Full License 


\section{Abstract}

\section{Background}

Glyphosate-based herbicides (GBHs) have previously been considered safe to humans. However, emerging evidence indicates that GBHs can disrupt the host microbiota and influence human health. To build upon our previous findings of gut dysbiosis and other adverse health effects resulting from lowdose exposure of GBHs (glyphosate and Roundup) in Sprague-Dawley rats, in particular pups during early development, we explore potential effects of GBHs on urinary metabolites and their interactions with gut microbiome in the same animal model.

\section{Methods}

Glyphosate and Roundup (equal molar for glyphosate) were administered in drinking water at the USA glyphosate ADI guideline $(1.75 \mathrm{mg} / \mathrm{kg}$ bw/day) to the dams (F0) starting from the gestational day (GD) 6 through the life-course of both dams and their pups (F1) (males and females). Urine samples were collected from F0 dams after pups' weaning and F1 pups at PND 70 and 125. The urine metabolites of 61 urine samples ( 14 from F0 dams and 30 from F1 pups) were profiled using non-targeted liquid chromatography - high resolution mass spectrometry (LC-HRMS). We further used supervised clustering analyses combined with random forest feature selection to evaluate differences in urine metabolome profiles between experimental groups.

\section{Results}

Overall urine metabolite profiles significantly differed between dams and pups and between female and male pups. When pups were stratified by sexes, Roundup and glyphosate exposure resulted in significant and distinctive changes in metabolite profiles. Specifically, both supervised clustering analysis and random forest feature selection methods identified a significant increase of homocysteine, a known risk factor of cardiovascular disease in both Roundup and glyphosate exposed pups, but in males only. Correlation network analysis between gut microbiome and urine metabolome pointed to Prevotella, a commensal genus with higher loading in male pups, to be negatively correlated with the level of homocysteine.

\section{Conclusions}

This study provides initial evidence that exposures to commonly used $\mathrm{GBH}$, at a currently acceptable human exposure dose, is capable of modifying the urine metabolites in both rat adults and pups. The strong link between Prevotella-Homocysteine suggests the potential role of GBHs in modifying the susceptibility of homocysteine related diseases like cardiovascular disease or inflammation through commensal microbiome.

\section{Background}


Glyphosate-based herbicides (GBHs), such as Roundup, are currently the most widely used herbicides in the world. GBHs are complex proprietary mixtures, with glyphosate as the main active ingredient. Since glyphosate was first produced in 1974 approximately 9.4 million tons of GBHs have been sprayed globally, nearly half a pound of glyphosate on every cultivated acre of land ${ }^{1}$. After the introduction of genetically modified organisms (GMOs) in 1996, in particular, glyphosate resistant crops, the global usage of GBHs has been increasing exponentially with about two-thirds of the total GBHs (by mass) sprayed in just the last decade. Besides GMO application, GBHs are also applied on non-GMO crops as desiccant in order to dry down crops and accelerate the harvest ${ }^{2}$. This practice further increases dietary exposure to glyphosate and its major metabolite aminomethylphosphonic acid (AMPA) ${ }^{3}$. Glyphosate mode of action is the inhibition of 5-enolpyruvylshikimate-3-phosphate synthase (EPSPS), involved in the synthesis of three aromatic amino acids: tyrosine, tryptophan, and phenylalanine ${ }^{4}$. As this shikimate pathway only exists in bacteria, fungi, and plants, but not in vertebrates, glyphosate was thought to impose minimal risks to mammals including humans. However, current emerging evidence suggest that glyphosate or GBH such as Roundup, can adversely affect mammalian biology via multiple mechanisms ${ }^{5-8}$. Several studies have also suggested the possible link between GBHs exposure and abnormality in neurodevelopment ${ }^{55,56}$. Among those mechanisms, it has been reported that GBHs exposure can alter the microbiota in honeybees, rats and other animals $\mathrm{s}^{9,10}$ and recently a shotgun metagenomic approach revealed that glyphosate alters the gut microbiome of Sprague-Dawley (SD) rats by inhibiting the shikimate pathway ${ }^{11}$. The gut microbiome is known to be a key player in modulating host metabolism; with multiple studies describing strong correlations between gut microbiome (microbial composition and metabolic function) and host metabolites ${ }^{12-15}$. Several microbial species and microbial metabolic functions have also been suggested to play a leading role in the gut-systemic metabolic interplays ${ }^{14,16}$. Therefore, we speculate that GBHs exposure may have the potential to modify the human microbiota, and which, in turn, to influence host metabolic functions.

Metabolomics is a promising approach to study the associations between environmental exposures and health effects ${ }^{17,18}$. Application of metabolomics profiling can potentially yield novel biomarkers, and inform molecular alterations underlying the toxicities. Over the last few years, many toxicological studies have demonstrated that metabolomics is a highly sensitive method in detecting effects associated with environmental exposures, where metabolite perturbations often happened prior to the histopathological changes ${ }^{16,19,20}$. However, up to date, few studies have been carried out on the effect of glyphosate exposure on host metabolomics profiles. In particular, study has neither used metabolomics to investigate the biological perturbations of glyphosate exposure nor investigated the interactions between GBH altered microbiome and metabolomics in early developmental stages, a critical period of susceptibility as denoted in the Developmental Origins of Health and Disease (DOHaD) paradigm. Therefore, in this pilot study, we performed untargeted metabolomics profiling to examine whether exposure to $\mathrm{GBH}$ at low doses comparable to those ubiquitously exposed to humans can affect urinary metabolomics profiles during early development. We further used paired urine metabolomics and gut 
microbiome data to test whether the $\mathrm{GBH}$ altered metabolites correlate with changes in the gut microbiome.

\section{Methods}

\section{Animals}

All animal study procedures were performed at the Cesare Maltoni Cancer Research Centre/Ramazzini Institute (CMCRC/RI) (Bentivoglio, Italy), and the animal experiment conducted with strict adherence to the Italian law regulating the use and treatment of animals for scientific purposes (Decreto legislativo $\mathrm{N}$. 26, 2014. Attuazione della direttiva n. 2010/63/UE in materia di protezione degli animali utilizzati a fini scientifici. - G.U. Serie Generale, n. 61 del 14 Marzo 2014). Before starting, the protocol was examined by the Internal Ethical Committee for approval. The protocol of the experiment was also approved and formally authorized by the ad hoc commission of the Italian Ministry of Health (ministerial approval $\mathrm{n}$. 710/ 2015-PR). As previously described ${ }^{21}$, all the pups were housed with their dam until weaning; then, separated into treatment groups and identified by ear punch. The animals were randomized in the different groups of treatment in order to have minimal differences in body weight among them, with a standard deviation of no more than $10 \%$ from the average. They were housed in Makrolon cages $(\mathrm{cm} 41$ $\times 25 \times 15)$ at two or three per cage, with a stainless-steel wire top and a shallow layer of white firewood shavings as bedding. All animals were kept in a single room at $23 \pm 3{ }^{\circ} \mathrm{C}$ and at $40-60 \%$ relative humidity with light/dark cycles at $12 \mathrm{~h}$ each using artificial light. The animals were given the same standard "Corticella" pellet diet (Piccioni Laboratory, Milan, Italy) for both breeders and offspring; both feed and tap water were available ad libitum. Feed and tap water were routinely analyzed to exclude biological and chemical contamination (mycotoxins, pesticides, arsenic, lead, mercury, selenium).

\section{Treatment}

The timeline of the experimental animal treatment and sample collection has been described previously ${ }^{9,21}$. As illustrated in Figure 1, we randomly selected 14 dams ( $N=5$ controls, $N=5$ glyphosate and $\mathrm{N}=4$ Roundup) and $30 \mathrm{~F} 1$ pups ( 15 female and 15 male). The F0 dams received the treatment through drinking water from gestation day (GD) 6 to the end of lactation (totally they were exposed for 49 \pm 2 days). The F1 pups received the treatment from their dams starting from in utero (GD 6) and mainly through milk during lactation. After weaning, F1 pups were treated through drinking water until sacrifice (PND 70 or PND 125).

\section{Urine and fecal sample collection}

Urine samples were collected at the end of lactation for dams and at PND70 and PND125 for pups. The urine samples were centrifuged to remove any debris $\left(50,000 \times \mathrm{g}\right.$ at $10^{\circ} \mathrm{C}$ for 15 minutes) then transferred to $1.5 \mathrm{ml}$ cryovials. About 2-3 fecal droppings from each pup were collected as described previously ${ }^{9}$. Briefly, forceps used for collecting droppings were washed and cleaned using sterile water and $1 \%$ sodium bicarbonate between each sampling to avoid cross contamination. The urine and fecal cryovials 
were stored at $-20^{\circ} \mathrm{C}$ until shipment on dry ice to the testing laboratories at Icahn School of Medicine at Mount Sinai.

Metabolomics analysis

Urine samples were thawed on ice, vortexed and diluted with water down to a specific gravity of 1.002 for pre-acquisition normalization. A $20 \mathrm{ul}$ aliquot of the diluted sample was prepared and stored at $-80^{\circ} \mathrm{C}$ until metabolomics analysis. Immediately prior to liquid chromatography - high resolution mass spectrometry (LC-HRMS) analysis, urine samples were combined with 180 ul of acetonitrile containing internal standards to remove proteins, and the supernatant transferred to LC vials.

Sample extracts were analyzed in ZIC HILIC positive (ZHP) and RP negative (RPN) modes separately using an ultra-high performance liquid chromatography (UHPLC) 1290 Infinity II system (including $0.3 \mu \mathrm{m}$ inline filter, Agilent Technologies, Santa Clara, USA) with 1260 Infinity II isocratic pump (including 1:100 splitter) coupled to a 6550 iFunnel or 6545 quadrupole-time time of flight (Q-TOF) mass spectrometer with a dual AJS electrospray ionization source (Agilent Technologies, Santa Clara, USA). Samples were maintained at $5^{\circ} \mathrm{C}$ in the autosampler module. For polar metabolites separation, $2 \mathrm{uL}$ of sample was injected onto a HILIC SeQuant ${ }^{\circledR}$ ZIC $®$-HILIC column $(100 \mathrm{~mm} \times 2.1 \mathrm{~mm}, 100 \AA$, $3.5 \mu \mathrm{m}$ particle size, Merck, Darmstadt, Germany) maintained at $25^{\circ} \mathrm{C}$. While for nonpolar metabolites separation, $2 \mathrm{uL}$ of sample sandwiched between $10 \mathrm{uL}$ of water was injected onto a Zorbax Eclipse Plus C18, RRHD column ( $50 \mathrm{~mm} \times 2.1 \mathrm{~mm}, 1.8 \mu \mathrm{m}$ particle size, Agilent Technologies, Santa Clara, USA) coupled to a guard column ( $5 \mathrm{~mm} \times 2 \mathrm{~mm}, 1.8 \mu \mathrm{m}$ Agilent Technologies, Santa Clara, USA) maintained at $50^{\circ} \mathrm{C}$. Samples were analyzed in randomized order. To monitor system stability, a pooled QC sample prepared by combining aliquots of all samples was injected routinely throughout the run. See Supplemental Information for further details.

\section{LC-MS pre-processing}

Suspect screening was performed using an in-house database of over 600 authentic standards analyzed under the same conditions. For untargeted data analysis, the raw data files were first converted into $\mathrm{mzxml}^{22}$ format and a peak table generated using $[\mathrm{XCMS}]^{23}$ with parameters optimized by [IPO] ${ }^{24}$. Metabolite features with a $\mathrm{CV}<30 \%$ in the pooled-QC injections and with a mean fold change $>3$ or $>1.5$ compared to blank extracts for untargeted analysis or suspect screening, respectively, were retained for further analysis. The metabolomics data was further imputed by k-nearest neighbor imputation using [knn_impute] with a cutoff $=0.4(40 \%$ missing values $)$ and normalized by [normalize_met] with default settings using the $\mathrm{R}$ package [MetaboDiff ${ }^{25}$.

\section{Statistical analysis}

Differential metabolomic analysis was performed using R package [MetaboDiff] ${ }^{25}$. Unsupervised principal component analysis (PCA) was performed to compare overall metabolomic profiles by age, sex and exposure types. Tests for statistical significance were performed with the PERMANOVA test (adonis 
function in the vegan R package ${ }^{26}$. Supervised partial least squares discriminant analysis (PLS-DA) was performed using the mixOmics $\mathrm{R}$ package ${ }^{27}$ to select the major contributing metabolomic features that differentiate between study groups; A PLS-DA Variable Importance in Projection (VIP) score >2.0 was used as the cutoff value to identify top features contributing to metabolic differences. For each selected feature, we also calculated the fold change ratio and compared the mean by non-parametric test. Alternatively, we used the random forest algorithm, a supervised machine learning approach, using $\mathrm{R}$ package [Boruta] ${ }^{28}$ to identify significant differential metabolomic features associated with exposures. The spearman correlation networks between microbiome and metabolites were constructed using $\mathrm{R}$ package [igrapgh $]^{29}$ and FDR adjusted p-values were obtained using R package [qvalue] ${ }^{30}$. The microbiome data of pups at PND125 was obtained from our previous study ${ }^{9}$.

\section{Results}

\section{Metabolomic profile between sex and adulthood groups}

After filtering, untargeted metabolomic profiling resulted in 4637 peaks in RPN and 5346 peaks in ZHP for further statistical analysis. An unsupervised principal components analysis was initially employed to compare the overall metabolomic profiles and detect outliers. We found the overall metabolomic profiles differed significantly between dams and pups, and between male and female pups, but not by exposure group (Glyphosate, Roundup or control) ( $P$-values $=0.001,0.001$ and 0.17 , respectively by multivariate PERMANOVA test, Figure $2 \mathrm{~A}$ and supplementary Figure 1$)$.

\section{Metabolomic features by exposure group and sex}

For the discrimination of the exposure types, the Partial Least Squares Discriminant Analysis (PLS-DA), a supervised clustering method was used (Figure 2B) using the 154 metabolites identified through suspect screening. Metabolomic features identified in sets with different exposure groups (Glyphosate, Roundup or non-exposed control) in dams and female or male pups were selected by using the cutoff of PLS-DA Variable Importance in Projection (VIP) score $>2.0$ and a P-value $<0.05$ in the metabolite fold change level. The full list of metabolites with VIP scores was listed in Table S1 and the selected features were shown in Table 1-3. Distinct differential metabolites by exposure types were found in both dams and pups. In dams (Table 1), glyphosate-exposed animals had significantly reduced methionine levels $(P$ value $=0.0079)$ compared to controls. Glyphosate-exposed dams also showed significant difference in 2Methylglutarate, Pipecolate, Riboflavin, Dimethylglycine and Beta-alanine methyl ester compared to Roundup $^{\text {TM. }}$. In female pups (Table 2), six metabolites (sebacic acid, N-methylglutamate, buberate, 10hydroxydecanoate, dodecanedioic acid and aminocaproate) were significantly different in glyphosateexposed compared to controls; whereas three metabolites (Adenine, $\mathrm{N}$-acetylglycine, L-proline) were different in Roundup exposed animals compared to controls. In addition, 5-Aminopentanoate was different between Glyphosate and Roundup exposed female pups. Of note, there was a trend of increased urinary adenine in both glyphosate and Roundup exposed female pups, however the VIP score was $>2$ only in the Roundup versus control comparison. In male pups (Table 3), four metabolites (1- 
aminocyclopropanecarboxylate, homocysteine, mevalolactone and 2-oxobutanoate) were significantly associated with Glyphosate exposure, whereas Roundup ${ }^{\mathrm{TM}}$ exposure resulted in dysregulation of homocysteine, phenylethanolamine, 2-oxobutanoate and biopterin. Importantly, compared to nonexposed male controls, both glyphosate and Roundup ${ }^{\text {TM }}$ exposed animals displayed a significant increase in urinary homocysteine levels. Between glyphosate and Roundup ${ }^{\mathrm{TM}}$ exposed male pups, we found 7 metabolites, including Phenylacetate, 3-Hydroxybenzoate, Aminocaproate, 10-Hydroxydecanoate, Guanosine, Glycocholate and Adenine were differentially expressed. We did not observe overlapping features shared between dams and pups or between female and male pups.

Besides of PLS-DA, we also performed the metabolomic features selection using a random forest (RF) machine learning feature selection method with the Boruta algorithm. Among the features selected by RF (Table S2), we found many selected features were consistent with the results from the PLS-DA.

\section{Correlations between metabolomics and microbiota}

To test whether top metabolites selected by PLS-DA in this study are linked to gut microbiota, we performed a correlation based network analysis between differential metabolites (VIP>2) in pups at PND125 and paired gut microbial composition from the same animal at PND125. Overall results from the metabolite-microbial correlation analyses are presented in Figure 3A. We found the Prevotella genus was strongly correlated with 10-hydroxydecanoate ( $\mathrm{rho}=0.57$, FDR adjusted $\mathrm{p}$-value $=0.015$ ), dodecanedioic acid (rho $=-0.58$, FDR adjusted $p$-value $=0.012$ ) and homocysteine (rho $=-0.64$, FDR adjusted $p$ value $=0.0028$ ). It should be noted that urinary homocysteine levels are also negatively correlated with not only Prevotella genus but also with its phylum, class, order and family. Similarly, the level of 10hydroxydecanoate is positively correlated with not only Prevotella genus but also its belonged Bacteroidetes phylum, Bacteroidia class, Bacteroidales order and Prevotellaceae family. In sex-stratified analyses (Figure 3B), male pups showed a clear inverse relationship between the levels of homocysteine and relative abundance of Prevotella; female pups showed a similar trend; however the changes did not reach statistical significance.

\section{Discussion}

GBHs are the most applied herbicides worldwide and humans are commonly exposed to these environmental chemicals at various doses. Environmental GBHs are ubiquitous and GBHs residues can be found in food ${ }^{31}$, drinking-water ${ }^{32}$, crops $^{33}$, animal feed ${ }^{34}$, groundwater ${ }^{3}$, even in air ${ }^{35}$. Although the effects of GBHs on human health are under intense public debate, evidence is emerging that they impact many health outcomes, including developmental and reproductive toxicity ${ }^{36-38}$, endocrine disruption 39,40 , host immunity ${ }^{41-43}$, obesity and diabetes ${ }^{7,44}$, gastrointestinal disorders ${ }^{45}$, cardiovascular disorders 46,47 and central nervous system dysfunction such as learning and memory impairment ${ }^{48}$, anxiety, depression ${ }^{49}$ and autism ${ }^{8}$. These chronic health outcomes may occur even at doses lower than established risk safety guidelines, in particular during critical development windows as denoted in the $\mathrm{DOHaD}$ paradigm ${ }^{50}$.Environmental exposures may lead to changes in metabolism ${ }^{18,51}$. A 
comprehensive, unbiased metabolite profiling, so-called "metabolomics" is a promising approach to study the associations between environmental exposures and health effects. Although our sample size was small, our results showed that gestational and early-life low-dose exposure to glyphosate or Roundup, significantly altered urine metabolomics, in both dams and offspring.

The one-carbon metabolism is a metabolic process that serves to activate and transfer $1 \mathrm{C}$ units for biosynthetic processes including purine and thymidine synthesis and homocysteine remethylation ${ }^{52}$. Folate is the essential cofactor in the one-carbon cycle, animals and humans cannot biosynthesize folate, thus requiring dietary intake or absorption of biosynthesized by gut microbiota ${ }^{53}$. In this study, we observed that low-dose GBH exposure can influence multiple metabolites involved in one-carbon metabolism. One key metabolite induced by GBHs exposure in male pups is homocysteine, a nonproteinogenic a-amino acid, biosynthesized from methionine that can be remethylated back into methionine or converted into cysteine with the aid of certain B-vitamins. Homocysteine metabolism of is highly dependent on vitamin derived cofactors; deficiencies in vitamin B12, folic acid and vitamin B6 are associated with higher levels of homocysteine in blood(hyperhomocysteinemia). In addition to homocysteine, we also observed GBH-induced deregulation of L-methionine and $\mathrm{N}$-methylglutamate also involved in one carbon metabolism. Interestingly, probiotic bacteria, including Prevotella using products of the shikimate pathway, which is inhibited by GBHs, can biosynthesize B vitamins, including folate ${ }^{54}$. Thus, it is plausible that the increased urine homocysteine we observed in male pups exposed to lowdosage GBHs results from reduced production of folic acid by Prevotella bacteria, paralleling the increased in homocysteine in dietary vitamin deficiencies.

Although the potential mechanism is still not clear, studies found that children with autism spectrum disorder (ASD) lack microbial diversity and have a decreased abundance in probiotics including Prevotella, potentially leading to reduced folate production by microbiota in individuals with $\mathrm{ASD}^{57}$. As our results indicate the role of GBHs in the folate deficiencies, we hypothesize that the environmental exposure to GBHs during early development may contribute to the ASD or other neurodevelopmental disorders through the depletion of beneficial folate-synthesizing enteric bacteria leading to the accumulation of homocysteine, a known neurotoxin which plays a role in brain damage, cognitive and memory decline ${ }^{58-61}$.

To be noted is that distinct metabolomic features were not only found between exposed and nonexposed, but also found between Glyphosate and Roundup exposed animals. Previous experimental evidence $^{62-64}$ supports that the Glyphosate formulations like Roundup are more toxic than Glyphosate alone, however the underlining mechanisms are still not clear. Our results suggested that metabolite profiling might be useful to identify possible metabolic pathways and to explain the excessive toxicity in those formulations.

This study has its limitations, mainly due to its small sample size, thus statistical power was limited subgroup analyses. Secondly, urinary creatinine concentrations are known to be different among age groups. Furthermore, the microbial survey using 16S rRNA gene amplicons-sequencing techniques in our 
study cannot capture the full metabolic activity of the microbial features correlated with host metabolomics. A more comprehensive whole genome metagenomic sequencing approach may be required for a full spectrum microbial metabolic function profiling to find underline mechanistic link between gut microbiome and the host metabolism.

In conclusion, to our knowledge, this is the first study on GBH-induced metabolomic changes at doses currently considered safe in humans. Metabolomic analyses revealed dynamic metabolic changes during the early developmental stage, a critical window of susceptibility. The strong link between PrevotellaHomocysteine suggests the potential role of commensal microbiome in modulating the metabolic alteration by GBH exposure. Further studies with a larger sample size, especially for subgroup analysis, are needed to investigate any underlying mechanistic associations more comprehensively.

\section{Declarations}

\section{Ethical Approval and Consent to participate}

The animal protocol was examined by the Internal Ethical Committee for approval. The protocol of the experiment was also approved and formally authorized by the ad hoc commission of the Italian Ministry of Health (ministerial approval n. 710/ 2015-PR)

\section{Consent for publication}

Not applicable

\section{Availability of supporting data}

16S rRNA gene sequencing information has been deposited into EMBL Nucleotide Sequence Database (ENA) and can be publicly accessed at www.ebi.ac.uk/ena/data/view/PRJEB12306

\section{Competing interests}

The authors declare that they have no competing interests.

\section{Funding}

This work was funded by Institution fund of Ramazzini Institute, Bologna, Italy (FB), MSSM seed fund (JH). NIEHS funded P30ES023515(JC, JP) and R01 ES029212 (JC).

\section{Authors' contributions}

$\mathrm{JH}, \mathrm{CL}, \mathrm{JC}$, and FB conceived and designed the study. JH, CL, FM, SP, DM, FB and JC managed data and sample collection and sample processing. FM, SP, DM and FB designed and conducted animal experiments. CL, MY, LP and JC designed and conducted metabolomic-profiling experiments. JH, CL, MY, 
$\mathrm{LP}$ and $\mathrm{JC}$ generated and analyzed data. $\mathrm{JH}, \mathrm{CL}, \mathrm{MY}$ and $\mathrm{JC}$ wrote the manuscript. All authors read the manuscript and provided critical comments.

\section{Acknowledgement}

We thank Georgia Dolios from the Senator Frank R. Lautenberg Environmental Health Sciences Laboratory at Icahn School of Medicine at Mount Sinai for her assistance with the the untargeted metabolomics data acquisition.

\section{References}

1. Benbrook CM. Trends in glyphosate herbicide use in the United States and globally. Environ Sci Eur. 2016; PMID: 27752438

2. Mills PJ, Kania-Korwel I, Fagan J, McEvoy LK, Laughlin GA, Barrett-Connor E. Excretion of the herbicide glyphosate in older adults between 1993 and 2016. JAMA - Journal of the American Medical Association. 2017.

3. Battaglin WA, Meyer MT, Kuivila KM, Dietze JE. Glyphosate and its degradation product AMPA occur frequently and widely in U.S. soils, surface water, groundwater, and precipitation. J Am Water Resour Assoc. 2014;

4. STEINRÜCKEN HC, AMRHEIN N. 5-Enolpyruvylshikimate-3-phosphate synthase of Klebsiella pneumoniae 2. Inhibition by glyphosate [N-(phosphononmethyl)glycine]. Eur J Biochem. 1984;

5. Cattani D, de Liz Oliveira Cavalli VL, Heinz Rieg CE, Domingues JT, Dal-Cim T, Tasca Cl, Mena Barreto Silva FR, Zamoner A. Mechanisms underlying the neurotoxicity induced by glyphosate-based herbicide in immature rat hippocampus: Involvement of glutamate excitotoxicity. Toxicology. 2014;

6. Tarazona J V., Court-Marques D, Tiramani M, Reich H, Pfeil R, Istace F, Crivellente F. Glyphosate toxicity and carcinogenicity: a review of the scientific basis of the European Union assessment and its differences with IARC. Archives of Toxicology. 2017.

7. Samsel A, Seneff S. Glyphosate's Suppression of Cytochrome P450 Enzymes and Amino Acid Biosynthesis by the Gut Microbiome: Pathways to Modern Diseases. Entropy. 2013;

8. Beecham JE, Seneff S. Is there a link between autism and glyphosate-formulated herbicides? J Autism. 2016;

9. Mao Q, Manservisi F, Panzacchi S, Mandrioli D, Menghetti I, Vornoli A, Bua L, Falcioni L, Lesseur C, Chen J, Belpoggi F, Hu J. The Ramazzini Institute 13-week pilot study on glyphosate and Roundup administered at human-equivalent dose to Sprague Dawley rats: Effects on the microbiome. Environ Heal A Glob Access Sci Source. 2018;17(1).

10. Motta EVS, Raymann K, Moran NA. Glyphosate perturbs the gut microbiota of honey bees. Proc Natl Acad Sci. 2018;

11. Mesnage R, Teixeira M, Mandrioli D, Falcioni L, Ducarmon QR, Zwittink RD, Amiel C, Panoff J-M, Belpoggi F, Antoniou MN. Shotgun metagenomics and metabolomics reveal glyphosate alters the gut 
microbiome of Sprague-Dawley rats by inhibiting the shikimate pathway. bioRxiv [Internet]. 2019 Jan 1;870105. Available from: http://biorxiv.org/content/early/2019/12/11/870105.abstract

12. Cani P, Delzenne N. The Role of the Gut Microbiota in Energy Metabolism and Metabolic Disease. Curr Pharm Des. 2009; PMID: 19442172

13. Nieuwdorp M, Gilijamse PW, Pai N, Kaplan LM. Role of the microbiome in energy regulation and metabolism. Gastroenterology. 2014;

14. Kovatcheva-Datchary P, Nilsson A, Akrami R, Lee YS, De Vadder F, Arora T, Hallen A, Martens E, Björck I, Bäckhed F. Dietary Fiber-Induced Improvement in Glucose Metabolism Is Associated with Increased Abundance of Prevotella. Cell Metab. 2015;

15. Fabbiano S, Suárez-Zamorano N, Chevalier C, Lazarević V, Kieser S, Rigo D, Leo S, Veyrat-Durebex C, Gaïa N, Maresca M, Merkler D, Gomez de Agüero M, Macpherson A, Schrenzel J, Trajkovski M. Functional Gut Microbiota Remodeling Contributes to the Caloric Restriction-Induced Metabolic Improvements. Cell Metab. 2018; PMID: 30174308

16. Zhao YY, Wang HL, Cheng XL, Wei F, Bai X, Lin RC, Vaziri ND. Metabolomics analysis reveals the association between lipid abnormalities and oxidative stress, inflammation, fibrosis, and Nrf2 dysfunction in aristolochic acid-induced nephropathy. Sci Rep. 2015;

17. Bundy JG, Davey MP, Viant MR. Environmental metabolomics: A critical review and future perspectives. Metabolomics. 2009.

18. Lankadurai BP, Nagato EG, Simpson MJ. Environmental metabolomics: An emerging approach to study organism responses to environmental stressors. Environmental Reviews. 2013.

19. Johnson $\mathrm{CH}$, Ivanisevic J, Siuzdak G. Metabolomics: Beyond biomarkers and towards mechanisms. Nature Reviews Molecular Cell Biology. 2016. PMID: 26979502

20. Chang H, Meng HY, Liu SM, Wang Y, Yang XX, Lu F, Wang HY. Identification of key metabolic changes during liver fibrosis progression in rats using a urine and serum metabolomics approach. Sci Rep. 2017;

21. Panzacchi S, Mandrioli D, Manservisi F, Bua L, Falcioni L, Spinaci M, Galeati G, Dinelli G, Miglio R, Mantovani A, Lorenzetti S, Hu J, Chen J, Perry MJ, Landrigan PJ, Belpoggi F. The Ramazzini Institute 13-week study on glyphosate-based herbicides at human-equivalent dose in Sprague Dawley rats: Study design and first in-life endpoints evaluation. Environ Heal A Glob Access Sci Source. 2018; PMID: 29843719

22. Chambers MC, MacLean B, Burke R, Amodei D, Ruderman DL, Neumann S, Gatto L, Fischer B, Pratt B, Egertson J, Hoff K, Kessner D, Tasman N, Shulman N, Frewen B, Baker TA, Brusniak MY, Paulse C, Creasy D, Flashner L, Kani K, Moulding C, Seymour SL, Nuwaysir LM, Lefebvre B, Kuhlmann F, Roark J, Rainer P, Detlev S, Hemenway T, Huhmer A, Langridge J, Connolly B, Chadick T, Holly K, Eckels J, Deutsch EW, Moritz RL, Katz JE, Agus DB, MacCoss M, Tabb DL, Mallick P. A cross-platform toolkit for mass spectrometry and proteomics. Nature Biotechnology. 2012.

23. Smith CA, Want EJ, O'Maille G, Abagyan R, Siuzdak G. XCMS: Processing mass spectrometry data for metabolite profiling using nonlinear peak alignment, matching, and identification. Anal Chem. 
2006;

24. Libiseller G, Dvorzak M, Kleb U, Gander E, Eisenberg T, Madeo F, Neumann S, Trausinger G, Sinner F, Pieber T, Magnes C. IPO: A tool for automated optimization of XCMS parameters. BMC Bioinformatics. 2015; PMID: 25888443

25. Mock A, Warta R, Dettling S, Brors B, Jäger D, Herold-Mende C. MetaboDiff: An R package for differential metabolomic analysis. Bioinformatics. 2018; PMID: 29718102

26. Oksanen J. Multivariate analysis of ecological communities in R: vegan tutorial. R Doc. 2015;43.

27. Rohart F, Gautier B, Singh A, Lê Cao KA. mixOmics: An R package for 'omics feature selection and multiple data integration. PLoS Comput Biol. 2017; PMID: 29099853

28. Kursa MB, Rudnicki WR. Feature selection with the boruta package. J Stat Softw. 2010;

29. Csárdi G, Nepusz T. The igraph software package for complex network research. InterJournal Complex Syst. 2006;1695:1-9. PMID: 1420

30. Storey J, Bass A, Dabney A, Robinson D. qvalue: Q-value estimation for false discovery rate control. R Packag version 2120 [Internet]. 2015; Available from: http://github.com/jdstorey/qvalue.

31. Granby K, Johannesen S, Vahl M. Analysis of glyphosate residues in cereals using liquid chromatography-mass spectrometry (LC-MS/MS). Food Addit Contam. 2003;

32. Quality D. Glyphosate and AMPA in Drinking-water. America (NY). 2005;

33. Duke SO. Glyphosate degradation in glyphosate-resistant and -susceptible crops and weeds. J Agric Food Chem. 2011;

34. Zhao J, Pacenka S, Wu J, Richards BK, Steenhuis T, Simpson K, Hay AG. Detection of glyphosate residues in companion animal feeds. Environ Pollut. 2018;

35. Chang F chih, Simcik MF, Capel PD. Occurrence and fate of the herbicide glyphosate and its degradate aminomethylphosphonic acid in the atmosphere. Environ Toxicol Chem. 2011;

36. Dai P, Hu P, Tang J, Li Y, Li C. Effect of glyphosate on reproductive organs in male rat. Acta Histochem. 2016;

37. Uren Webster TM, Laing L V., Florance H, Santos EM. Effects of glyphosate and its formulation, roundup, on reproduction in zebrafish (Danio rerio). Environ Sci Technol. 2014;

38. De Liz Oliveira Cavalli VL, Cattani D, Heinz Rieg CE, Pierozan P, Zanatta L, Benedetti Parisotto E, Wilhelm Filho D, Mena Barreto Silva FR, Pessoa-Pureur R, Zamoner A. Roundup disrupts male reproductive functions by triggering calcium-mediated cell death in rat testis and Sertoli cells. Free Radic Biol Med. 2013;

39. Drašar P, Poc P, Stárka L. Glyphosate, an important endocrine disruptor. Diabetol Metab Endokrinol Vyziv. 2018;

40. Varayoud J, Durando M, Ramos JG, Milesi MM, Ingaramo PI, Muñoz-de-Toro M, Luque EH. Effects of a glyphosate-based herbicide on the uterus of adult ovariectomized rats. Environ Toxicol. 2017;

41. Ma J, Li X. Alteration in the cytokine levels and histopathological damage in common carp induced by glyphosate. Chemosphere. 2015; 
42. Kreutz LC, Gil Barcellos LJ, de Faria Valle S, de Oliveira Silva T, Anziliero D, Davi dos Santos E, Pivato M, Zanatta R. Altered hematological and immunological parameters in silver catfish (Rhamdia quelen) following short term exposure to sublethal concentration of glyphosate. Fish Shellfish Immunol. 2011;

43. Hong Y, Yang X, Yan G, Huang Y, Zuo F, Shen Y, Ding Y, Cheng Y. Effects of glyphosate on immune responses and haemocyte DNA damage of Chinese mitten crab, Eriocheir sinensis. Fish Shellfish Immunol. 2017;

44. De Long NE, Holloway AC. Early-life chemical exposures and risk of metabolic syndrome. Diabetes, Metabolic Syndrome and Obesity: Targets and Therapy. 2017.

45. D’Brant J. GMOs, Gut Flora, the Shíkimate Pathway and Cytochrome Dysregulation. Nutr Perspect J Counc Nutr. 2014;

46. Paumgartten FJR. Glyphosate exposure, cardiovascular diseases and cancer risks. J Public Heal Emerg. 2017;

47. Gress S, Lemoine S, Séralini GE, Puddu PE. Glyphosate-Based Herbicides Potently Affect Cardiovascular System in Mammals: Review of the Literature. Cardiovasc Toxicol. 2015;

48. Gallegos CE, Baier CJ, Bartos M, Bras C, Domínguez S, Mónaco N, Gumilar F, Giménez MS, Minetti A. Perinatal Glyphosate-Based Herbicide Exposure in Rats Alters Brain Antioxidant Status, Glutamate and Acetylcholine Metabolism and Affects Recognition Memory. Neurotox Res. 2018;

49. Aitbali Y, Ba-M'hamed S, Elhidar N, Nafis A, Soraa N, Bennis M. Glyphosate based-herbicide exposure affects gut microbiota, anxiety and depression-like behaviors in mice. Neurotoxicol Teratol. 2018;

50. Heindel JJ, Balbus J, Birnbaum L, Brune-Drisse MN, Grandjean P, Gray K, Landrigan PJ, Sly PD, Suk W, Slechta DC, Thompson C, Hanson M. Developmental origins of health and disease: Integrating environmental influences. Endocrinology. 2015.

51. Noecker C, Chiu H-C, McNally CP, Borenstein E. Defining and Evaluating Microbial Contributions to Metabolite Variation in Microbiome-Metabolome Association Studies. bioRxiv. 2018;

52. Ducker GS, Rabinowitz JD. One-Carbon Metabolism in Health and Disease. Cell Metabolism. 2017.

53. Engevik MA, Morra CN, Röth D, Engevik K, Spinler JK, Devaraj S, Crawford SE, Estes MK, Kalkum M, Versalovic J. Microbial Metabolic Capacity for Intestinal Folate Production and Modulation of Host Folate Receptors. Front Microbiol. 2019;

54. Yoshii K, Hosomi K, Sawane K, Kunisawa J. Metabolism of dietary and microbial vitamin b family in the regulation of host immunity. Frontiers in Nutrition. 2019.

55. Seneff S, Nigh GL. Evidence that Glyphosate Disrupts Neurodevelopment Glyphosate and Anencephaly: Death by A Thousand Cuts. J Neurol Neurobiol. 2017;

56. McVey KA, Snapp IB, Johnson MB, Negga R, Pressley AS, Fitsanakis VA. Exposure of C. elegans eggs to a glyphosate-containing herbicide leads to abnormal neuronal morphology. Neurotoxicol Teratol. 2016; 
57. Slattery J, Macfabe DF, Frye RE. The Significance of the Enteric Microbiome on the Development of Childhood Disease: A Review of Prebiotic and Probiotic Therapies in Disorders of Childhood. Clin Med Insights Pediatr. 2016;

58. Seshadri S. Elevated plasma homocysteine levels: Risk factor or risk marker for the development of dementia and Alzheimer's disease? Journal of Alzheimer's Disease. 2006.

59. Chigurupati S, Wei Z, Belal C, Vandermey M, Kyriazis GA, Arumugam T V., Chan SL. The homocysteine-inducible endoplasmic reticulum stress protein counteracts calcium store depletion and induction of CCAAT enhancer-binding protein homologous protein in a neurotoxin model of Parkinson Disease. J Biol Chem. 2009;

60. Tjiattas L, Ortiz DO, Dhivant S, Mitton K, Rogers E, Shea TB. Folate deficiency and homocysteine induce toxicity in cultured dorsal root ganglion neurons via cytosolic calcium accumulation. Aging Cell. 2004;

61. Seshadri S, Wolf PA. Homocysteine and the brain: Vascular risk factor or neurotoxin? Lancet Neurology. 2003.

62. Mesnage R, Defarge N, Spiroux De Vendômois J, Séralini GE. Major pesticides are more toxic to human cells than their declared active principles. Biomed Res Int. 2014; PMID: 24719846

63. Vanlaeys A, Dubuisson F, Seralini GE, Travert C. Formulants of glyphosate-based herbicides have more deleterious impact than glyphosate on TM4 Sertoli cells. Toxicol Vitr. 2018;

64. Lee HL, Kan CD, Tsai CL, Liou MJ, Guo HR. Comparative effects of the formulation of glyphosatesurfactant herbicides on hemodynamics in swine. Clin Toxicol. 2009;

\section{Figures}

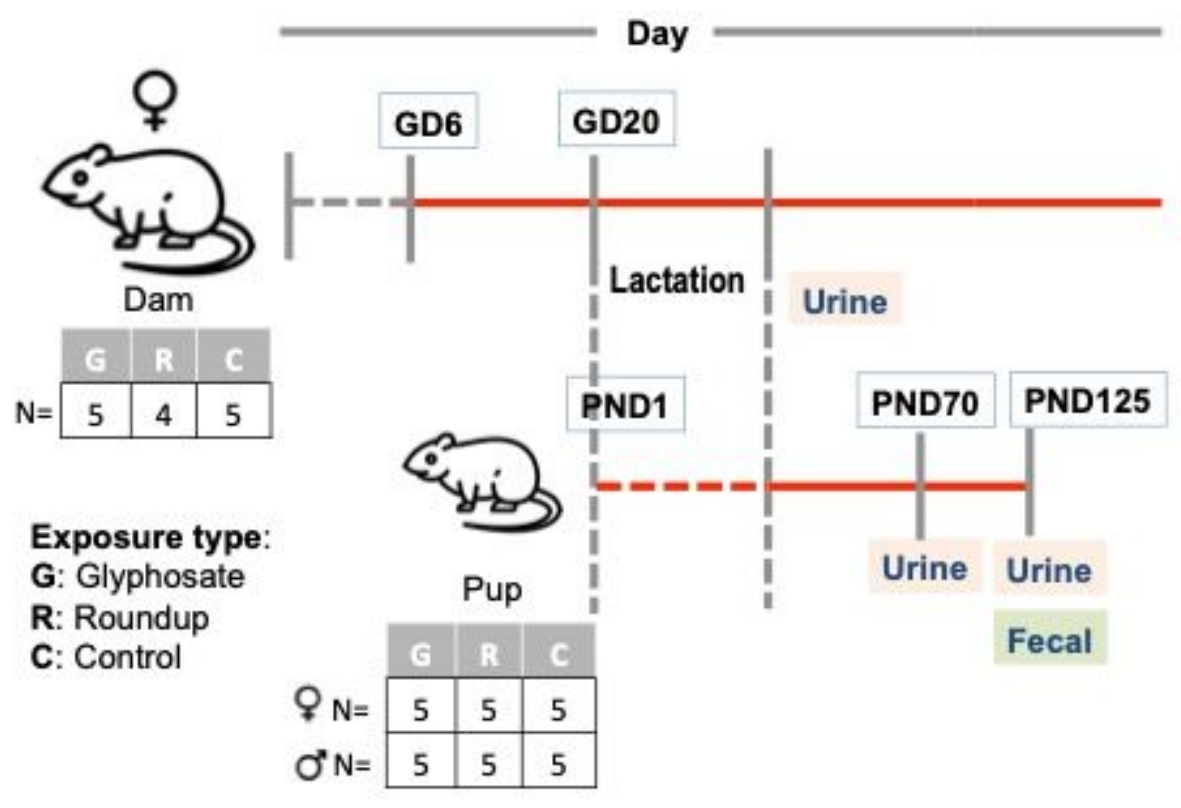

Figure 1 
Study design. Test compounds including pure glyphosate and Roundup ${ }^{\mathrm{TM}}$ are administered ad libitum in drinking water. Dams are treated through drinking water starting from the gestation day 6th (GD 6). Pups are treated from embryonic life (GD 6) indirectly from dams milk following birth (GD20) until the end of lactation, then directly through drinking water for 90 days after weaning (until PND 125). Urine samples are collected after lactation from dams and PND70 and PND 125 from pups. Fecal samples are collected at PND 125 from pups.

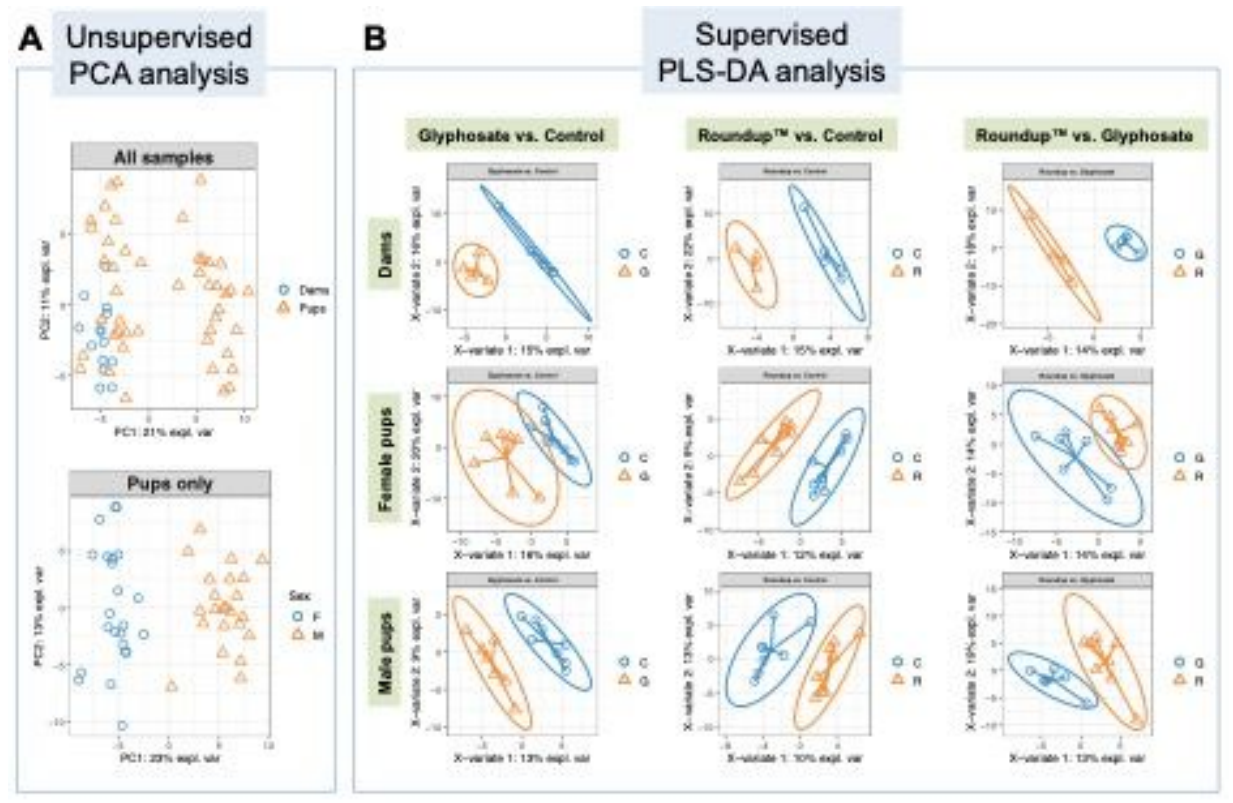

\section{Figure 2}

Differential metabolomic profiles by glyphosate exposure in rat female and male pups. 1A. Unsupervised PCA analysis showed the metabolomic profiles were differentiated by adulthood and sex. 1B. Supervised Partial Least Squares Discriminant Analysis was performed and showed distinct metabolome clusters that are strongly associated with the exposure chemicals among groups by adulthood and sex. 


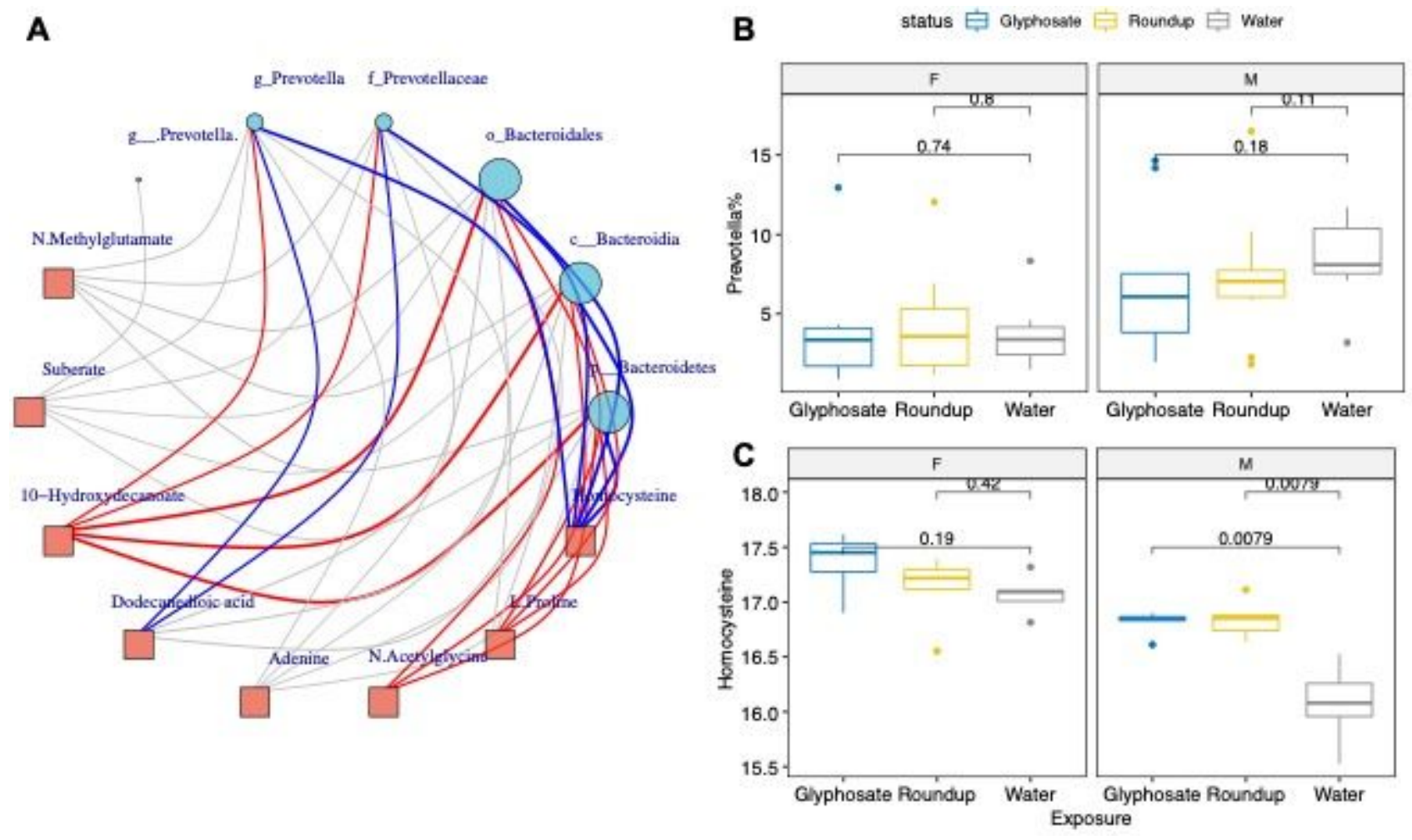

Figure 3

The urine homocysteine levels in pups were associated with gender and glyphosate or Roundup ${ }^{\mathrm{TM}}$ exposure and were strongly correlated with Prevotella abundance in gut microbiota. 2A. Correlation network between exposure associated metabolic features and gut microbiota. Prevotella and its belonged Bacteroidetes phylum to Prevotellaceae family all showed strong negative correlation with the urine Homocysteine. The links with FDR adjusted p-value $<0.05$ were colored with red (positive correlation) and blue (negative correlation). 2B and 2C. Boxplots showed that Prevotella abundances were lower in female pups than male pups. Prevotella was reduced in exposed male pups. In contrast, the female pups have higher Homocysteine than the male pups and the homocysteine levels were significantly increased by exposure in male pups.

\section{Supplementary Files}

This is a list of supplementary files associated with this preprint. Click to download.

- Tables.pdf

- tables1.csv

- tables4.csv

- tables2.csv

- Supplementarydata.docx

- tables3.csv 